\title{
Galactic-center S stars as a prospective test of the Einstein equivalence principle
}

\author{
Angélil, R ; Saha, P
}

\begin{abstract}
The S stars in the Galactic-center region are found to be on near-perfect Keplerian orbits around presumably a supermassive black hole, with periods of 15-50 yr. Since these stars reach a few percent of light speed at pericenter, various relativistic effects are expected and have been discussed in the literature. We argue that an elegant test of the Einstein equivalence principle should be possible with existing instruments, through spectroscopic monitoring of an S star concentrated during the months around pericenter, supplemented with an already-adequate astrometric determination of the inclination. In essence, the spectrum of an S star can be considered a heterogeneous ensemble of clocks in a freely falling frame, which near pericenter is moving at relativistic speeds.
\end{abstract}

DOI: https://doi.org/10.1088/2041-8205/734/1/L19

Posted at the Zurich Open Repository and Archive, University of Zurich ZORA URL: https://doi.org/10.5167/uzh-48282

Journal Article

Published Version

Originally published at:

Angélil, R; Saha, P (2011). Galactic-center S stars as a prospective test of the Einstein equivalence principle. Astrophysical Journal Letters, 734(1):L19.

DOI: https://doi.org/10.1088/2041-8205/734/1/L19 


\title{
GALACTIC-CENTER S STARS AS A PROSPECTIVE TEST OF THE EINSTEIN EQUIVALENCE PRINCIPLE
}

\author{
Raymond Angélil and Prasenjit Saha \\ Institute for Theoretical Physics, University of Zürich, Winterthurerstrasse 190, CH-8057 Zürich, Switzerland \\ Received 2011 January 31; accepted 2011 May 2; published 2011 May 20
}

\begin{abstract}
The S stars in the Galactic-center region are found to be on near-perfect Keplerian orbits around presumably a supermassive black hole, with periods of 15-50 yr. Since these stars reach a few percent of light speed at pericenter, various relativistic effects are expected and have been discussed in the literature. We argue that an elegant test of the Einstein equivalence principle should be possible with existing instruments, through spectroscopic monitoring of an S star concentrated during the months around pericenter, supplemented with an already-adequate astrometric determination of the inclination. In essence, the spectrum of an S star can be considered a heterogeneous ensemble of clocks in a freely falling frame, which near pericenter is moving at relativistic speeds.
\end{abstract}

Key words: Galaxy: center - relativistic processes

\section{INTRODUCTION}

The equivalence of gravitational and inertial mass, or that gravity can be canceled by transforming to a freely falling frame, was tested within classical celestial mechanics to high precision by the end of the nineteenth century. After all, if Mercury had a gravitational constant differing from (say) Jupiter's by one part per million, Mercury's orbit would not have fitted classical dynamics well enough to highlight the anomalous precession $\left(43^{\prime \prime}\right.$ per century, or $10^{-7}$ ) that was later explained by general relativity.

The Einstein equivalence principle (EEP) adds to the classical or weak equivalence principle the further physical postulate that special relativity holds locally in a freely falling frame. A consequence of the EEP is that the effects of gravity on test particles are fully described by endowing spacetime with a Riemannian metric, and having the particles follow geodesics of that metric. A further consequence of the EEP is that the temporal metric component is, to leading order, given by $g_{t t}=1+2 \Phi$, where $\Phi$ is the Newtonian potential. Hence, gravity causes time dilation.

Different aspects of the EEP have been verified by multiple experiments, as reviewed extensively by Will (2006). In particular, null-redshift experiments test that gravity-whatever it does-does the same to clocks of different types. Pound-Rebka experiments verify that, in a static gravitational field, time is dilated by a factor $g_{t t}^{-1 / 2} \simeq 1-\Phi$. GPS satellites and binary pulsars are effectively freely falling clocks moving at ${ }^{1} \sim 10^{-5}$ and $\sim 10^{-3}$, respectively, and exhibit time dilation of $g_{t t}^{-1} \simeq 1-2 \Phi$.

As excellent as the existing experiments on the EEP are, it would be even nicer to have a laboratory with experiments on multiple materials, the whole freely falling at relativistic speeds. In this Letter, we suggest that the S stars in the Galacticcenter region approximate such a laboratory. Stellar spectra contain absorption lines from different atomic species, which can be regarded as different clocks. The EEP asserts that special relativity holds in the star's frame for all atomic processes (local Lorentz invariance), irrespective of where the star is (local position invariance), and these can be tested by observations of a single star. The remaining ingredient of the EEP, namely, that a freely falling frame does not itself depend on the composition of the star (universality of free fall, UFF) requires multiple stars.

\footnotetext{
1 We use geometrized units: $G M=c=1$, where $M$ is the black hole mass.
}

The nature of a violation of the UFF is difficult to speculate on, however, one could imagine, for example, fits from different $\mathrm{S}$ stars' redshift curves all yielding different values for the black hole $(\mathrm{BH})$ mass. Such a deduction would signal a violation of the UFF.

Going beyond the EEP, yet another possibility is that a star with a relativistically significant gravitational self energy, i.e., a pulsar, may give different results. Such a result would be a violation of the strong equivalence principle. It is expected that a significant number of pulsars inhabit the inner milliparsec, and although none have yet been found, searches are currently underway (Macquart et al. 2010).

The $\mathrm{S}$ stars achieve the highest speeds of any known geodesics. For example, S2 reaches $v>0.03$ at pericenter, which it last passed in 2002 and will again in 2018. The pericenter speed is an order of magnitude faster than for known binary pulsars. The spatial scales at pericenter are of order a light day. Hence, even if the quantitative constraints are initially modest, the $\mathrm{S}$ stars would test the EEP at velocity and spatial scales not reached by other experiments.

Astrometric and redshift observations of the $\mathrm{S}$ stars show them to be on orbits (so far) indistinguishable from pure Keplerian (Gillessen et al. 2009a; Ghez et al. 2008; Gillessen et al. 2009b). Keplerian elements are known to high accuracy for many stars. For example, for $\mathrm{S} 2$ the orbital inclination is measured to be $135^{\circ} \pm 1^{\circ}$. Expected relativistic effects have been discussed extensively in the literature. These include not only time dilation (Zucker et al. 2006), but also secular orbit precession (Rubilar \& Eckart 2001; Will 2008) plus Newtonian perturbations from other stars (Merritt et al. 2010), kinematic effects due to space curvature and frame dragging (Kannan \& Saha 2009; Preto \& Saha 2009), and composite redshift perturbations including light-path effects (Angélil \& Saha 2010; Angélil et al. 2010).

The EEP implies a time dilation of $1+2 / r$. Hence, a spectral line intrinsically at $v_{0}$ will be redshifted to $v$, where

$$
\frac{v_{0}}{v}=\left(1+v_{\text {los }}\right)\left(1+\frac{2}{r}\right) \text {. }
$$

Redshift is conventionally defined as $v_{0} / v-1$. It is, however, somewhat more convenient if one defines $\ln \left(v_{0} / v\right)$ as the redshift, and we will do so. In any case, the $2 / r$ term constitutes a redshift perturbation of $\mathcal{O}\left(v^{2}\right)$ and is the strongest relativistic perturbation. In other words, the leading-order perturbation due to relativity is time dilation on a Keplerian orbit. Space curvature 
perturbs the redshift at $\mathcal{O}\left(v^{3}\right)$, frame dragging due to $\mathrm{BH}$ spin at $\mathcal{O}\left(v^{4}\right)$, with additional physical effects continuing at higher orders (Angélil et al. 2010). One way to test the EEP would be to replace $2 / r$ in Equation (1) by $\alpha / r$ and fit observed redshift curves for the parameter $\alpha$. In fact, this has already been attempted (Zucker et al. 2006) but the available data appeared not yet sufficiently accurate to overcome systematic uncertainties. We argue below that observations concentrated near pericenter will be particularly useful.

Relativistic perturbations to the $\mathrm{S}$ stars increase with decreasing pericenter distance. In orbit fitting from spectroscopy, as one looks to stars with longer periods, the perturbations to the redshift from relativity become overwhelmed by perturbations due to massive perturbers. Of all the compact objects orbiting the central BH, we see only the brightest stars. Thousands of solar masses worth of compact objects and a possibly significant dark matter component are expected to inhabit the inner arcseconds. Our lack of understanding of the form of this extended system may play a significant role in obscuring relativistic perturbations for large orbits. However, current models for the Newtonian perturbations are estimated to most likely be weaker than the time dilation effect for the shortest-period stars such as S2 (Schödel et al. 2009; Merritt et al. 2010). Hence, we will disregard the extended-mass component in our analysis.

\section{OBSERVABLES}

Consider a star on a pure Keplerian orbit. Elementary celestial mechanics gives the position of the star parametrically, that is, both coordinates and time are expressed as functions of the so-called eccentric anomaly $\psi$. At time

$$
t=\frac{P}{2 \pi}(\psi-e \sin \psi),
$$

the position of the star in its orbital plane is

$$
(x, y, z)=\frac{P}{2 \pi \sqrt{a}}\left(\cos \psi-e, \sqrt{1-e^{2}} \sin \psi, 0\right) .
$$

Here $P$ is the period, $e$ is the eccentricity, while $a$ is the semimajor axis in units of the gravitational radius of the $\mathrm{BH}$.

We now rotate the coordinate system, first by the argument of periapsis $\omega$ about the $z$-axis, then by the inclination $I$ about the new $x$-axis. The observer is now along the new $z$-axis. The redshift is

$$
\ln \frac{\nu_{0}}{v}=\frac{d z}{d t}+\frac{\alpha}{r}=\frac{A_{C} f(e, \omega, \psi)+A_{R}}{1-e \cos \psi},
$$

where

$$
f(e, \omega, \psi) \equiv \sqrt{1-e^{2}} \cos \omega \cos \psi-\sin \omega \sin \psi
$$

and we have introduced the coefficients

$$
A_{C} \equiv \frac{\sin I}{\sqrt{a}} \quad \text { and } \quad A_{R} \equiv \frac{\alpha}{a},
$$

which can be interpreted as the amplitudes of the classical and relativistic contributions to the redshift. We also note that $t$ above is the coordinate time of emission. The time of observation is, of course, $t$ plus the light travel time, whose varying part is the Rømer time delay, which is the $z$-coordinate of the star

$$
A_{C} \frac{P}{2 \pi}\left[(\cos \psi-e) \sin \omega+\sqrt{1-e^{2}} \sin \psi \cos \omega\right]
$$

and can also be interpreted as the time integral of the redshift.

Classically, the redshift curve determines only the combination $\sin I / \sqrt{a}$, leaving the inclination unknown. Hence, for a given redshift curve, the inferred orbital speed becomes infinite if the orbit is face-on. Not surprisingly, relativity prevents that happening. From Equations (4) and (6) it follows that

$$
\sin ^{2} I=\frac{A_{C}^{2}}{A_{R}} \alpha .
$$

Hence, time dilation breaks the inclination degeneracy if $\alpha$ is known, or allows $\alpha$ to be measured if $I$ is known from astrometry.

It is worth mentioning that another way in which relativity can break the inclination degeneracy is through the well-known precession of

$$
\Delta \omega=\frac{6 \pi}{a\left(1-e^{2}\right)}
$$

per orbit. For binary pulsars, the cumulative $\omega$ precession amounts to several degrees per year, allowing $a$ and hence $I$ to be inferred (Brumberg et al. 1975). For Galactic-center stars, however, the $\omega$ precession builds up much more slowly. Over a single orbit the redshift due to precession is $\sim \mathcal{O}\left(v^{3}\right)$ because $\Delta \omega \sim \mathcal{O}\left(v^{2}\right)$ over one orbit.

\section{PARAMETER RECOVERY}

We treat the following seven parameters as unknown.

1. The period $P$.

2. An additive constant on $t$.

3 . The eccentricity $e$.

4. The argument of pericenter $\omega$, which along with the inclination $I$ sets the orbit orientation with respect to the observer. Since the observer is on the $z$-axis, the nodal angle $\Omega$ corresponds to a rotation around the line of sight, which leaves the redshift curve invariant.

5. The intrinsic frequency $v_{0}$. This is the absolute line calibration plus any offset due to the observer's radial velocity. Changing $v_{0}$ shifts the redshift curve vertically. The tangential motion of the observer is neglected here, since the Sun's orbital speed of $\sim 200 \mathrm{~km} \mathrm{~s}^{-1}$ in the Galaxy would contribute no significant Doppler shift.

6. A classical redshift amplitude $A_{C}$.

7. A relativistic redshift amplitude $A_{R}$. In fact, $a, I, \alpha$ form a degenerate trio and are absorbed into the non-degenerate dummy parameters $A_{C}$ and $A_{R}$ via Equation (6).

Astrometry independently measures the first four of these, as well as I. Astrometric observations also involve six other parameters: the position and proper motion of the Galactic center on the sky, the distance to the Galactic center, and $\Omega$. The mass of the $\mathrm{BH}$ is not an independent parameter, since it is a function of $P$ and $a$. For our purposes, only $I$ from astrometry is essential. In testing the EEP, while we shall argue that observation of the star over a short time around pericenter suffices for spectroscopy, recovery of the inclination via astrometry can be done anywhere on the orbit.

It is important to note $v_{0}$ and $v$ need not refer to a single spectral line. The spectra of S stars (see, e.g., Martins et al. 2008) contain multiple features. Most are early-type stars with $\mathrm{H}$ and $\mathrm{He}$ features, while about $10 \%$ are late-type stars with molecular and metal bands/lines and little or no $\mathrm{H}$ or He. Hence, $\mathrm{S}$ star spectra could, in principle, test the equivalence principle 


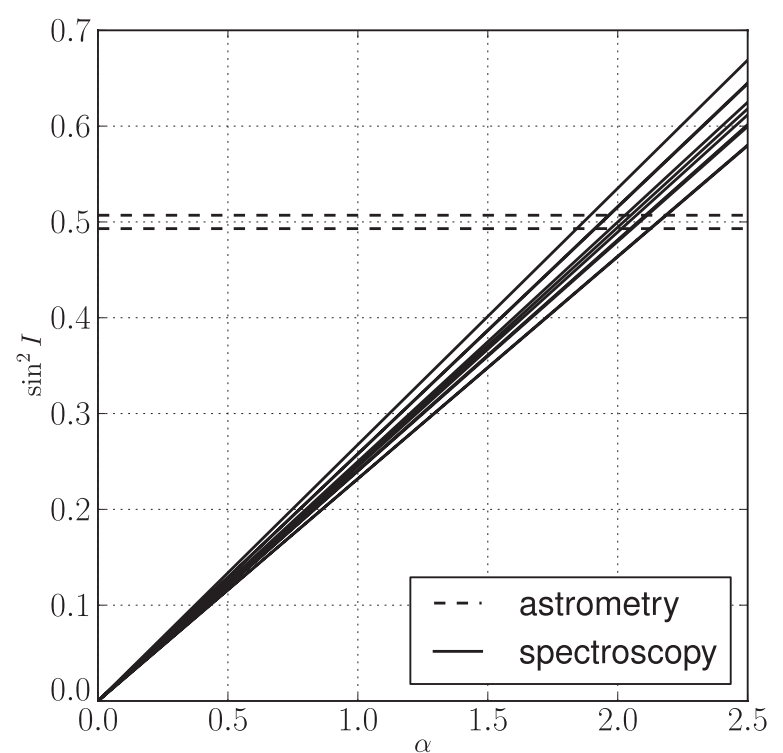

Figure 1. Recovery of $\alpha$ for 10 mock data sets. The ratio $A_{C}^{2} / A_{R}$ is the slope of the lines in the above plot and is recovered from mock spectroscopic data of 14 data points with accuracy $10 \mathrm{~km} \mathrm{~s}^{-1}$, concentrated around pericenter. The horizontal lines are the upper and lower confidence levels for the recovered inclination from astrometry, taken from Gillessen et al. (2009b). The intersection point corresponds to the value of $\alpha$ for which both data types agree on the inclination.

for multiple atomic processes. If the stellar atmosphere does not change appreciably over an orbit, an observed spectrum can be cross-correlated on a logarithmic wavelength scale with a spectrum observed at some other epoch, and the cross-correlation peak would directly give the redshift $\ln \left(v_{0} / v\right)$ with $v_{0}$ an unknown constant. If different atomic/molecular species behave differently in a freely falling frame, the shape of the crosscorrelation curve would change. Alternatively, multiple spectral features could be fitted simultaneously with variable redshift. We do not, however, attempt to model the observable spectra explicitly in this Letter.

We now simulate the recovery of $\alpha$ as follows. We generate 10 mock redshift data points of S2 taken over two months at pericenter, plus four additional data points, at $\pm 1, \pm 2 \mathrm{yr}$ around pericenter. The data are generated with $\alpha=2$ and orbital parameters taken from Gillessen et al. (2009b). To them we add Gaussian random noise at a dispersion of $10 \mathrm{~km} \mathrm{~s}^{-1}$ and then fit via the seven parameters. We then assume $I$ has been measured by astrometry and use Equation (8) to recover $\alpha$. Figure 1 shows an example for a few mock data realizations at a fixed accuracy and Figure 2 shows the dependency of the recovered value of alpha with the data accuracy.

\section{DISCUSSION}

Testing the equivalence principle using a combination of spectroscopy and astrometry seems possible in the near future. In comparing the spread in $A_{C}^{2} / A_{R}$ from mock data to the recovered value for $I$ from real astrometric data (illustrated in Figure 1), in testing the equivalence principle using S2, the current accuracy available from astrometry sits at a comfortable level. Spectroscopic accuracy of S2 at $10 \mathrm{~km} \mathrm{~s}^{-1}$ is not yet available, but seems plausible with future observations. For the late-type star S35, which has a more favorable spectrum, a fit error of $10 \mathrm{~km} \mathrm{~s}^{-1}$ has been achieved (Gillessen et al. 2009b). We remark that any systematic errors that do not change between observations are harmlessly absorbed into $v_{0}$.

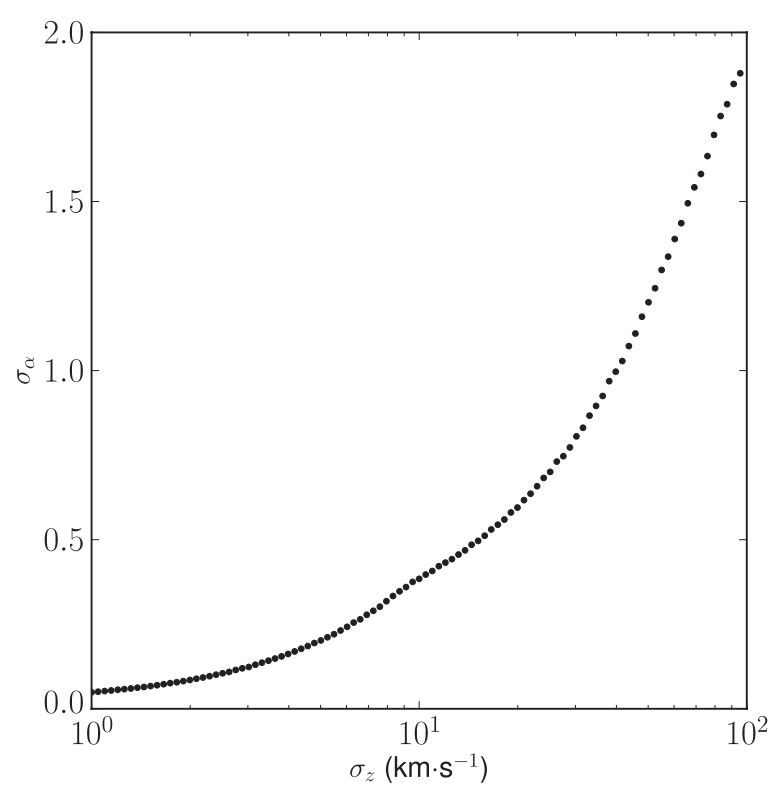

Figure 2. $1 \sigma$ level in the recovered value of $\alpha$ for spectroscopic data of different accuracy. At each data accuracy level, we have performed the same procedure illustrated in Figure 1, except that 60,000 mock data realizations have been used.

Naturally, in detecting relativistic effects on S stars, data during pericenter passage are of greatest value. With instrumentation currently available, an observation program concentrated over two months during S2's next pericenter passage (2018) will prove to be sufficient as a test for the EEP. Figure 2 argues that a small handful of spectral measurements of S2 at $10 \mathrm{~km} \mathrm{~s}^{-1}$ around pericenter imply a $1 \sigma$ accuracy on $\alpha$ of $\sim 0.3$.

The approach we have taken above focuses on the essentials. The degeneracy between $\alpha, I$, and $a$ for spectroscopy has been lifted by using astrometry only to provide $I$. In practice, all the parameters are fitted simultaneously to both astrometry and spectroscopy. We have done simulations to verify that when this is done, the degeneracy is implicitly broken by the mechanism highlighted in this Letter.

Relativistic effects can be expected to become increasingly important as corrections in other astrophysics relating to the $\mathrm{S}$ stars. Three areas where this can be expected are the following.

1. The combination of spectroscopic and astrometric $\mathrm{S}$ star data provides us with the distance to the center of the galaxy. Astrometry is sensitive to the angular size of the orbit, while spectroscopy on the physical size. The quotient is the distance to the galactic center (Eisenhauer et al. 2003).

2. The position of the observed line depends on the velocity of the BH-star system with respect to the Earth. Spectroscopy therefore has the power to determine our velocity with respect to the central BH (Angélil et al. 2010), thus constraining the $U$ component of the Galactic local standard of rest.

3. The form of the mass distribution within the inner arcsecond affects the S star orbits. A better understanding of the density profile will provide insight into the region's star capture and formation history, and to the central dark matter distribution (Ghez et al. 2008; Gillessen et al. 2009b).

In exploring (1) and (2), one cannot easily avoid relativity simply by considering stars with larger orbit sizes (3), as perturbations due to the enclosed mass become a problem. With the accuracy regime that spectroscopy will enter in the coming decade, one of two types of perturbations to the redshift must 
be faced: either those from the extended mass distribution, for $\mathrm{S}$ stars with large orbits, or, those perturbations from relativity, for smaller orbit $\mathrm{S}$ stars. While effects due to relativity are well understood in principle, and can be easily treated, the constitution of the extended system is poorly understood and requires a more grueling treatment. In the coming decade, spectroscopic $\mathrm{S}$ star accuracy at $\sim 10 \mathrm{~km} \mathrm{~s}^{-1}$ is expected to be available, and the discovery of stars closer in to the $\mathrm{BH}$ is anticipated. In light of these prospects, so that constraints on these quantities may be improved, relativistic perturbations, while interesting in their own right, can no longer be ignored and must be faced.

The authors thank S. Gillessen, G. F. R. Ellis, and J.-P. Uzan for discussion and comments.

\section{REFERENCES}

Angélil, R., \& Saha, P. 2010, ApJ, 711, 157

Angélil, R., Saha, P., \& Merritt, D. 2010, ApJ, 720, 1303
Brumberg, V. A., Zeldovich, I. B., Novikov, I. D., \& Shakura, N. I. 1975, Pis'ma Astron. Zh., 1, 5

Eisenhauer, F., Schödel, R., Genzel, R., Ott, T., Tecza, M., Abuter, R., Eckart, A., \& Alexander, T. 2003, ApJ, 597, L121

Ghez, A. M., et al. 2008, ApJ, 689, 1044

Gillessen, S., Eisenhauer, F., Fritz, T. K., Bartko, H., Dodds-Eden, K., Pfuhl, O., Ott, T., \& Genzel, R. 2009a, ApJ, 707, L114

Gillessen, S., Eisenhauer, F., Trippe, S., Alexander, T., Genzel, R., Martins, F., \& Ott, T. 2009b, ApJ, 692, 1075

Kannan, R., \& Saha, P. 2009, ApJ, 690, 1553

Macquart, J., Kanekar, N., Frail, D. A., \& Ransom, S. M. 2010, ApJ, 715, 939

Martins, F., Gillessen, S., Eisenhauer, F., Genzel, R., Ott, T., \& Trippe, S. 2008, ApJ, 672, L119

Merritt, D., Alexander, T., Mikkola, S., \& Will, C. M. 2010, Phys. Rev. D, 81, 062002

Preto, M., \& Saha, P. 2009, ApJ, 703, 1743

Rubilar, G. F., \& Eckart, A. 2001, A\&A, 374, 95

Schödel, R., Merritt, D., \& Eckart, A. 2009, A\&A, 502, 91

Will, C. M. 2006, Living Rev. Rel., 9, 3

Will, C. M. 2008, ApJ, 674, L25

Zucker, S., Alexander, T., Gillessen, S., Eisenhauer, F., \& Genzel, R. 2006, ApJ 639, L21 